\title{
Characterisation of Lead Barium Zirconate thin films for utilisation of the Electrocaloric Effect.
}

\author{
C.P. Shaw ${ }^{\mathrm{a}^{*}}$, Qi Zhang ${ }^{\mathrm{a}}$, T.M. Correia ${ }^{\mathrm{b}}$, P.M. Weaver ${ }^{\mathrm{c}}$
}

asurface Engineering and Nanotechnology Institute, Manufacturing and Materials

Department, Cranfield University, Cranfield, Bedfordshire. MK43 OAL, UK.

"c.p.shaw@cranfield.ac.uk , q.zhang@cranfield.ac.uk ("corresponding author)

${ }^{b}$ Knowledge Transfer Network. Tatiana.correia@ktn-uk.org

c InotekUK. paul.weaver@innotecuk.com

\begin{abstract}
Characterisation of a composition of Lead Barium Zirconate thin films deposited by a spin coating sol-gel process onto platinised silicon substrates has been undertaken to investigate a potential application utilising the reported electrocaloric properties of the material. Xray diffraction studies revealed that above $700^{\circ} \mathrm{C}$ the presence of pyrochlore phase was replaced by ferroelectric phase but the identification of antiferroelectric phases previously reported could not be identified. Wet etching of the films produced an insoluble residue containing only evidence of zirconium out of the original three metals, which resembled a skeletal structure similar to the original rosette morphology of the annealed films. Elemental analysis of the annealed films using EDX indicated a non-target $\mathrm{Pb}: \mathrm{Ba}: \mathrm{Zr}$ ratio, which suggested a reduced $\mathrm{Zr}$ content of the films. "Solution-like" rather than "sol-like" nature of the spin coated sol and phase boundary proximity are proposed for reported observations, and a mechanism is proposed to account for the unusual observed trend in dielectric constant.
\end{abstract}

Keywords

ceramics; thin films; electronic materials; crystallisation; ferroelectricity; nucleation.

\section{Introduction}

The electrocaloric effect is the adiabatic temperature change, or isothermal entropy change, caused by the polarisation change of a dielectric material when subjected to an external electric field. A recent review around potential electrocaloric materials [1] has highlighted the importance of ferroelectric materials, which demonstrate much larger electrocaloric effect (ECE) than traditional ceramic thin films, and which makes high performance solid-state cooling devices possible in the future. Measurements of ECE in thin films are mostly based on field-induced polarisation measurements and Maxwell relations to derive $\Delta S$ and $\Delta T$ when an applied field is altered[2-12]. Very limited studies on a direct method of measuring the ECE have concentrated on using a high resolution calorimeter approach to measure the temperature change[13]. To address this issue and obtain accurate measurement of the ECE, development of a Electrocaloric Effect(ECE) 
direct measurement method is a goal. One challenge is that the substrate under the film causes the fast heat dissipation, which increases the difficulty in measuring accurately the temperature change of the thin film caused by the application of an external electric field. Possibly, the formation of free standing or thin membrane supported ferroelectric materials can limit the heat loss/transfer mechanisms, both increasing the detectability of temperature change and simplifying the modelling of the structure. Published work by Saranaya[5] Mischenko[14] and Peng [15] represents some of the largest proposed $\Delta T$ values reported for the ECE to date, with the later [15] claiming changes of $45 \mathrm{~K}$ at $60 \mathrm{KV} / \mathrm{m}$ for Lead Barium Zirconate (PBZ), and importantly near room temperature. This present study reports on further work carried out on the synthesis and characterisation of PBZ thin films based on the promising composition for electrocaloric applications looked at in the Peng et al. [15] study, and highlights some of the processing issues in scale up prior to the development of free standing membrane devices.

\section{Material and Methods}

The target composition for finally annealed films was $\mathrm{Pb}_{0.8} \mathrm{Ba}_{0.2} \mathrm{ZrO}_{3}$ but a sol composition with $20 \%$ excess of lead based on this formula, was prepared to allow for lead loss during the film annealing. A $0.3 \mathrm{M}$ sol with the main solvent components (glacial acetic acid:water:2-methoxyethanol(2ME)) in a 1:1:1 volume ratio was prepared as follows.

First, $10.93 \mathrm{~g}$ of lead (II) acetate trihydrate (Sigma-Aldrich $99.5 \%$ ) and $1.53 \mathrm{~g}$ of barium acetate(Acros Organics 99+\%) were dissolved in a mixture $29.37 \mathrm{~g}$ of glacial acetic acid(Sigma-Aldrich $99 \%$ ) and $26.45 \mathrm{~g}$ of water, by stirring in a covered beaker for $1 \mathrm{hr}$ at room temperature.

$14.04 \mathrm{~g}$ of zirconium n-propoxide solution (Sigma Aldrich, 70\%in 1-propanol) was mixed with $27.02 \mathrm{~g}$ of 2-methoxyethanol (Sigma-Aldrich $99.8 \%$ ) in a $250 \mathrm{ml}$ round bottom flask under nitrogen using a glove box (Bassaire Ltd, Manchester). The sealed flask was removed from the glove box and a preweighed $3.3 \mathrm{~g}(10 \%$ excess relative to $\mathrm{Zr}$ ) of acetylacetone (Fluka $99.5 \%$ ) was quickly added to the solution and swirled to stabilise the metal organic. The stabilised solution was then stirred for 30 mins before combining with the $\mathrm{Pb} / \mathrm{Ba}$ solution, and the combined "Sol" solution was stirred for $24 \mathrm{hrs}$ at room temperature. The Sol was then filtered through a $0.45 \mu \mathrm{m}$ filter (Whatman Zap Cap ${ }^{\mathrm{TM}}$ ) and stored at room temperature before use. Particle size measurements were carried out using a Zetasizer ${ }^{\mathrm{TM}}$ (Model 3000, Malvern Ltd.), to investigate potential aging and potential shelf life of the "SOLs". For PBZ crystallization studies, electroded silicon wafers were prepared as substrates to allow subsequent electrical characterisation. Silicon wafers (Orientation: <100>. Resist: 10-30ohm-cm: Doping: P-type) with a thermal oxide coating were used supplied from Mi-Net Technology Ltd. (UK). Titanium/platinum (Ti/Pt) coatings were deposited by physical vapour deposition (PVD) using a Nordiko TM Ltd. sputtering system. This PVD approached used Rf (Titanium) and DC(Platinum) (300 W) water cooled, magnetron sputtering to deposit the bottom $\mathrm{Ti} / \mathrm{Pt}$ electrodes using Argon gas at $5 \mathrm{mT}$ and without substrate heating to achieve the target thicknesses of $10 \mathrm{~nm} / 100 \mathrm{~nm}$ respectively. 


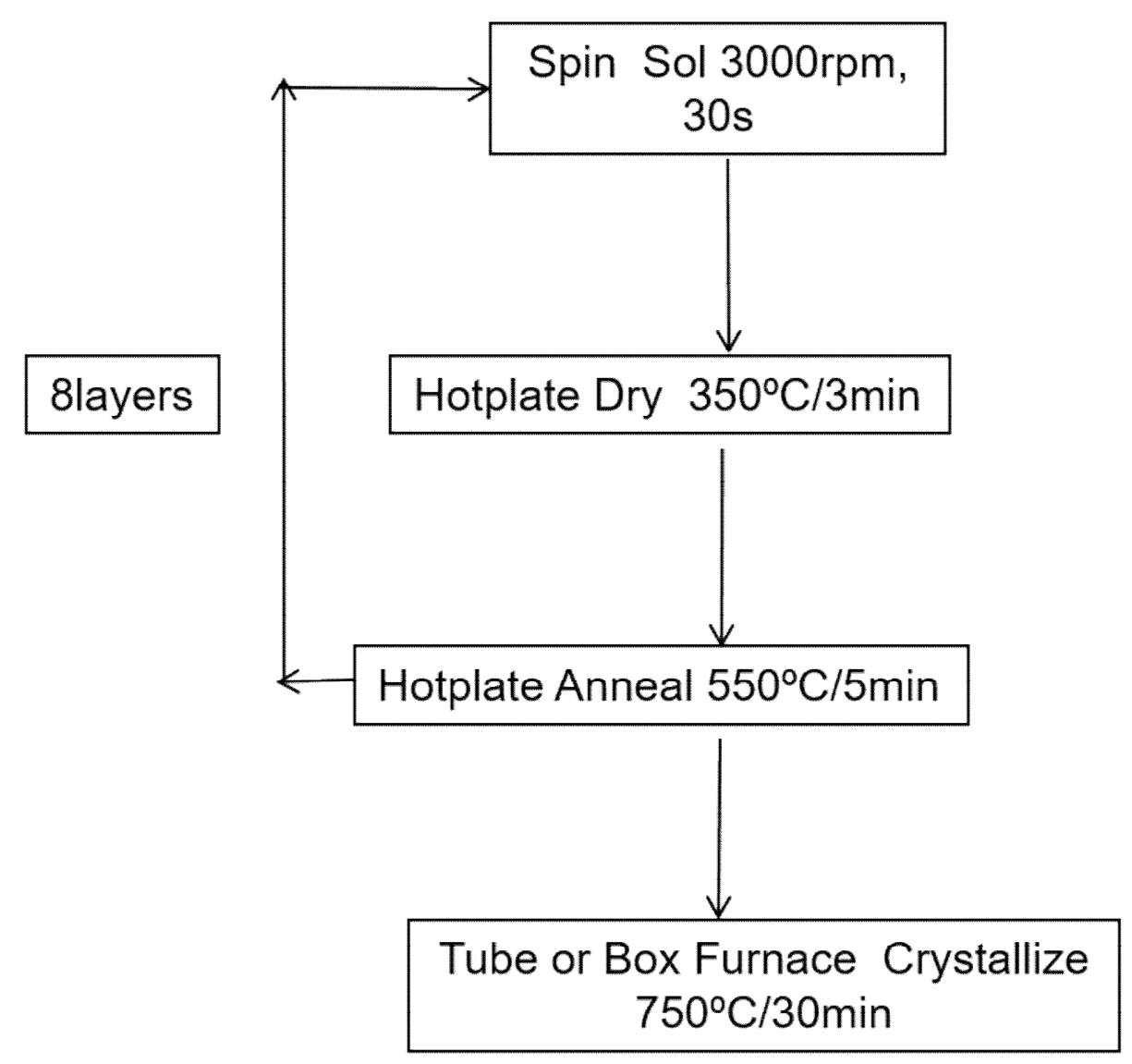

Figure 1. Sol-gel spin coating deposition cycle. Primary PBZ film deposition conditions based on previous study [15].

Figure 1 shows the deposition cycle used to deposit and build up film thickness of PBZ films as reported in the literature [15]. Pieces of Ti/Pt coated wafers $\left(\sim 2 \times 2 \mathrm{~cm}^{2}\right)$ were first dried on a hotplate at $100^{\circ} \mathrm{C}$. To aid wetting of the Pt surface by the sol, it was found that spin rinsing with distilled water and blow drying with nitrogen immediately before deposition of the first layer greatly improved the qualitative appearance of the deposited layer and subsequent film. The sol was applied using a syringe fitted with a $0.2 \mu \mathrm{m}$ filter $\left(\right.$ Whatman $^{\mathrm{TM}}$ ) to help reduce defects from particulate impurities. Following static sol application, a layer was coated using an spin coater (Electronic Micro Systems Ltd.) with an acceleration speed of $\sim 2000 \mathrm{rps}$, and then annealed using two hotplates (CEE 1100 Brewer Science, and Sawatec HP 160/700 Sawatzki Technology) respectively. This was repeated for a total of 8 layers, and then a single furnace crystallisation carried out. A small Carbolite ${ }^{\mathrm{TM}}$ tube furnace (Model: MTF 10/25/130) was initially used to try to replicate results obtained from the referenced work [15], but samples were also prepared in a Carbolite ${ }^{\mathrm{TM}}$ box furnace (Model: ELF 11/6) to accommodate the necessary scale up required to process a full wafer. A target thickness of $\sim 380 \mathrm{~nm}$ was expected for such an 8 layer film. Additional samples, looking at variations of the annealing and crystallization times were also made to try to help to solve some of the processing issues encountered. Film characterisation was carried out on annealed and part annealed samples using a Polyvar microscope (Reichert Jung), for optical studies, an X-Ray Diffraction system (Model: D500. Siemens Ltd. ) for crystallisation investigation and scanning 
electron microscopy (FEI XL30 SFEG) with EDX to complement optical studies and look for compositional variation. Dielectric measurements were also made using an impedance analyser (Wayne kerr Electronics Ltd. Model 3245)) for the purposes of this study to provide comparison with published values. Hysteresis measurements were collected from a RT66A (Radiant Technology Ltd.)

\section{Results}

Based on qualitative visual studies, the prepared PBZ sols were green solutions with good clarity even after 6 months. In initial work, a fine precipitate had been observed after trying to stabilize the Zirconium alkoxide using a 1:1 Zr/acetylacetone ratio, which was believed to be zirconium oxide, and due to incomplete transfer of all the acetylacetone. The subsequent addition of the $10 \%$ excess amount subsequently ensured at least an equimolar transfer, and the resultant clear "sols". Consequently, with no evidence of precipitation within these prepared sols, there is high confidence that the target composition of reactants was being deposited. Particle sizing results carried out on diluted samples of the sol over a 6 month period gave consistent values of $12 \pm 6 \mathrm{~nm}$. However, it is worth noting that the zetasizer indicated relatively low sampling counts suggesting that the measurement was operating in the "noise" region of the machine, and the evidence of the low counts is potentially an indication of a solution rather than a "sol" nature to the prepared sols.

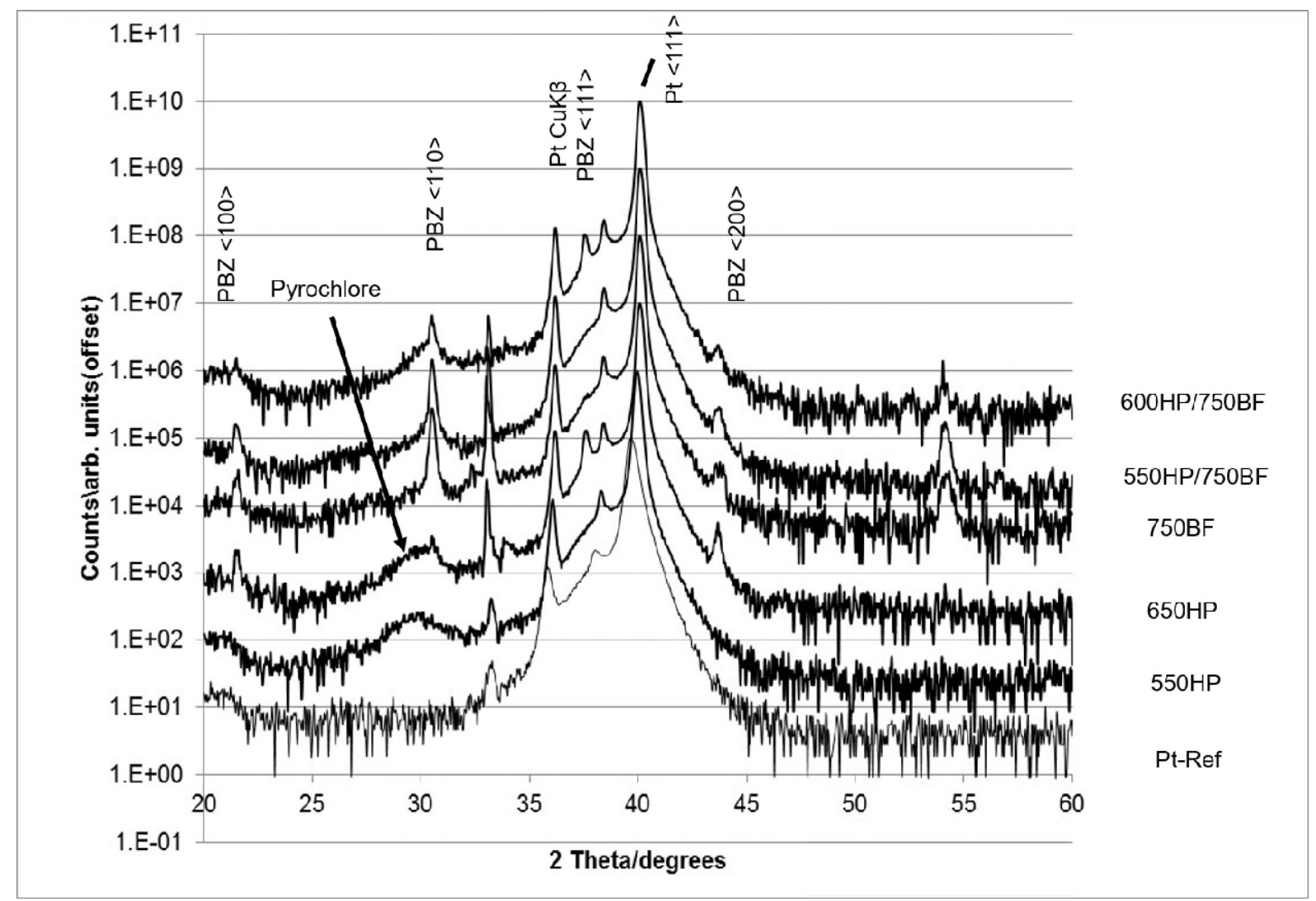

Figure 2. XRD comparison of PBZ four layer films. Films prepared under differing annealing conditions using hotplate (HP) and/or box furnace (BF), and including a non-annealed platinum reference (Pt-ref) for comparison. 
Figure 2 shows the initial XRD analysis on 4 layer samples crystallised with varying temperature, and clearly shows the removal of the broad pyrochlore phase peak centred at $29.84^{\circ}$, above $650{ }^{\circ} \mathrm{C}$, as the characteristic perovskite $<101 / 110>$ nature reflections at $30.48^{\circ}$ develops. Figures 3 and 4 compare the study made on the two furnace types used for the high temperature anneal step, for films made from two different sol batches. The most striking observation from the study which is best observed in figure 4 was the greater intensity of the peak at $37.48^{\circ}$ (typical of $\langle 111\rangle$ perovskite) for the samples annealed in the small tube furnace, and which was consistent for two different sol batches.

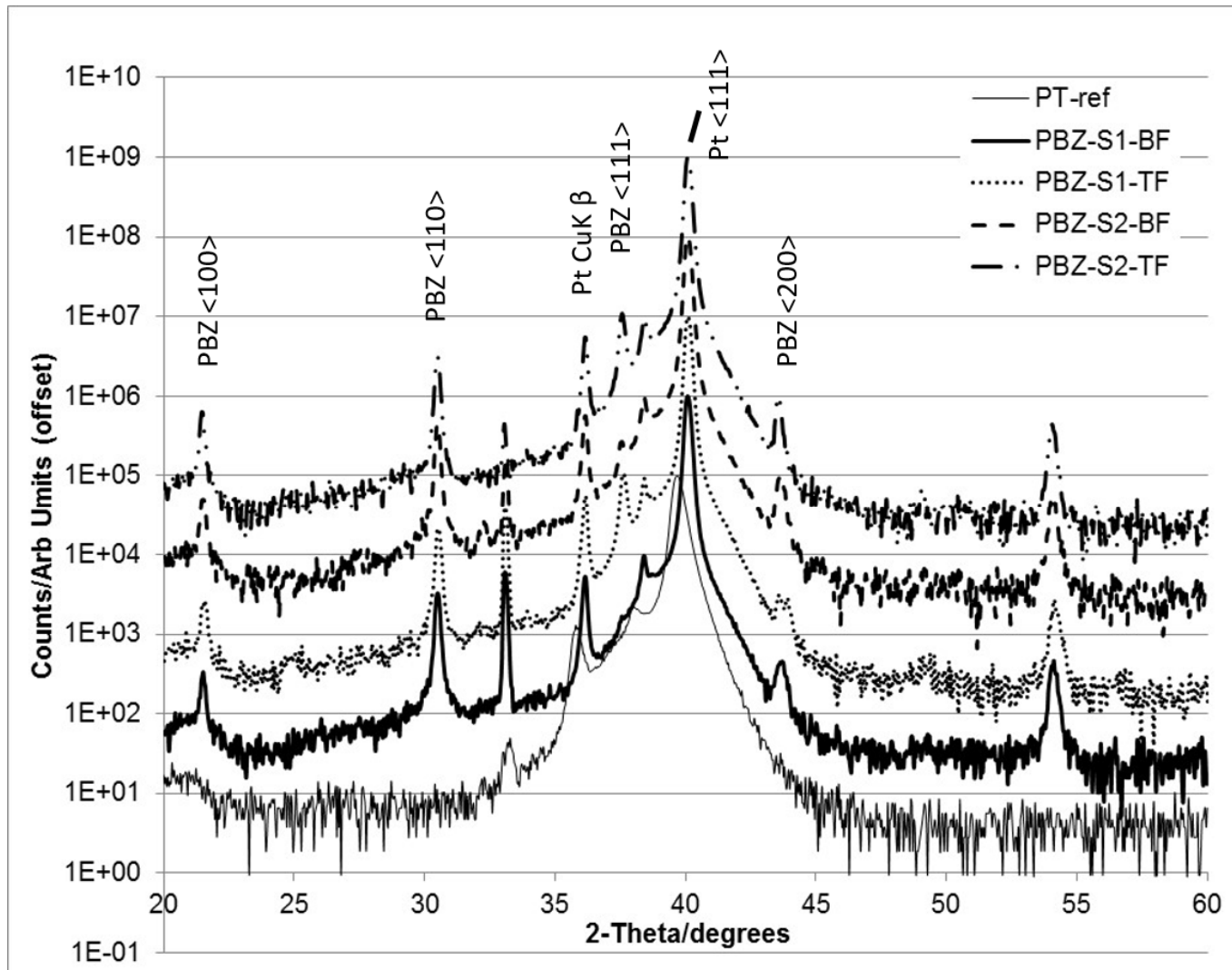

Figure 3. XRD comparison of PBZ films prepared form different sol batches in two different furnaces. Films have been prepared from two sols $(\mathrm{S} 1, \mathrm{~S} 2)$ using either a tube (TF) or box (BF) furnace. 


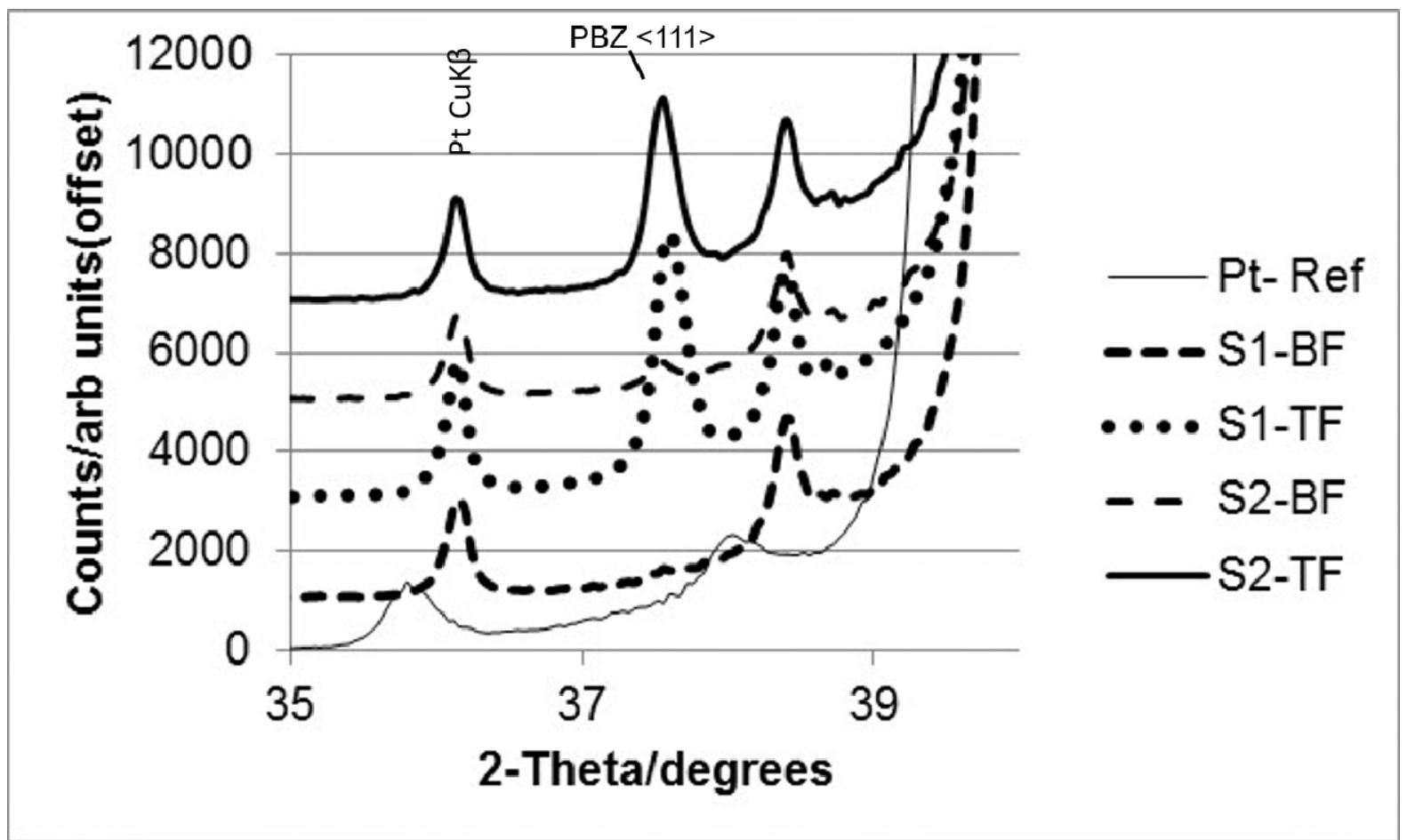

Figure 4. XRD comparison of PBZ films prepared form different sol batches in two different furnaces.(Enlarged area of figure 3).

In terms of film morphology figures $5 a-b$ and $6 a-b$ show images taken on the optical microscope. Figures $5 \mathrm{a}$ and $5 \mathrm{~b}$ are representative of the samples prepared based on the processing conditions in figure 1 and taken after the $550^{\circ} \mathrm{C}$ hotplate anneal (figure 5a) and after the furnace treatment (figure $5 \mathrm{~b}$ ) respectively. Subsequent wet etching of the films to carry out film thickness measurements and electrical characterisation, left unusual residual deposits which are shown in figures $6 a$ and $6 \mathrm{~b}$ and figure 7 shows the XRD comparison for an annealed and etched PBZ sample.

a)

b)

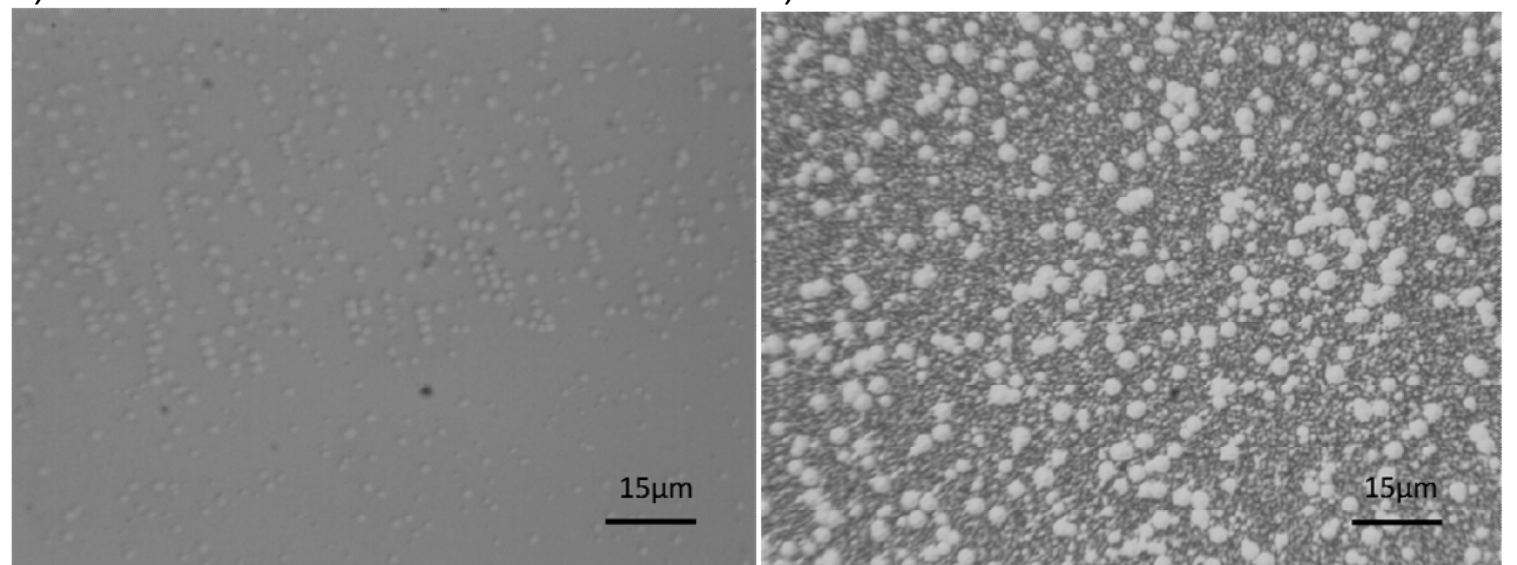

Figure 5. Optical images of PBZ annealed films. a) After $550^{\circ} \mathrm{C}$ hotplate anneal. b) After $550^{\circ} \mathrm{C}$ hotplate $/ 750^{\circ}$ furnace anneal. 
a)

b)

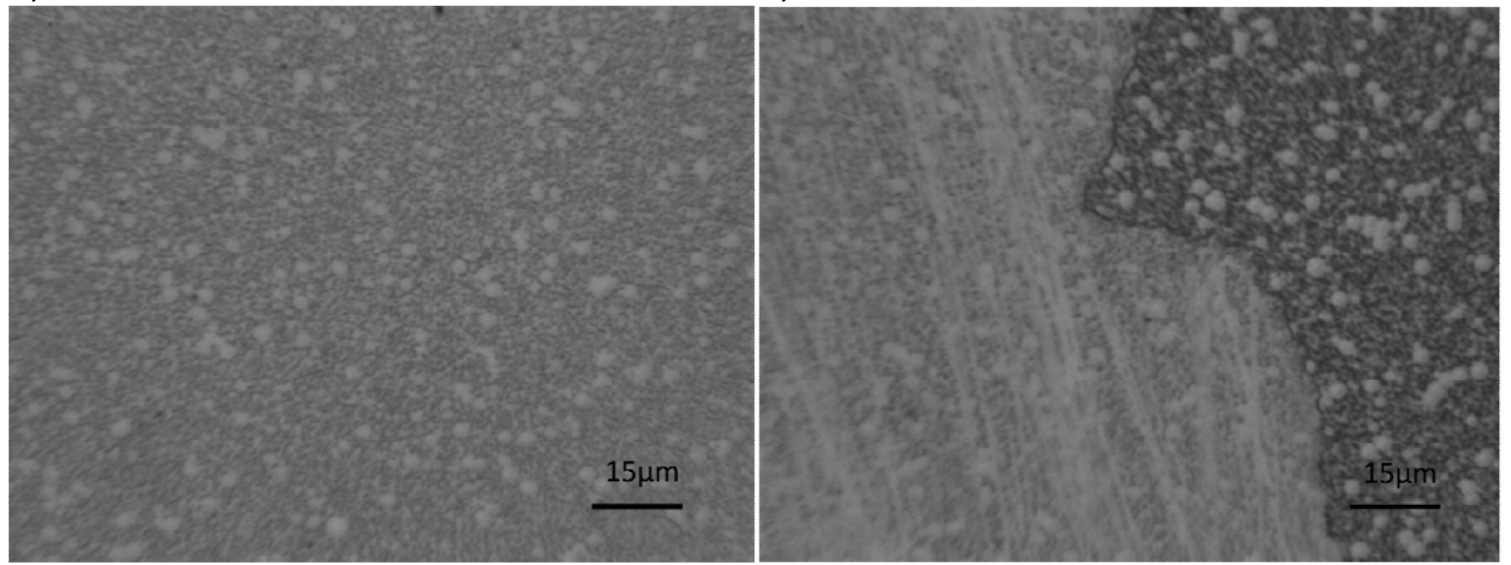

Figure 6. Optical image of residual film left after $\mathrm{HF} / \mathrm{HNO} 3$ wet etch. a) Fully exposed area. b) Area where PBZ film was masked. (visible smearing of residue in etched region in (b).)

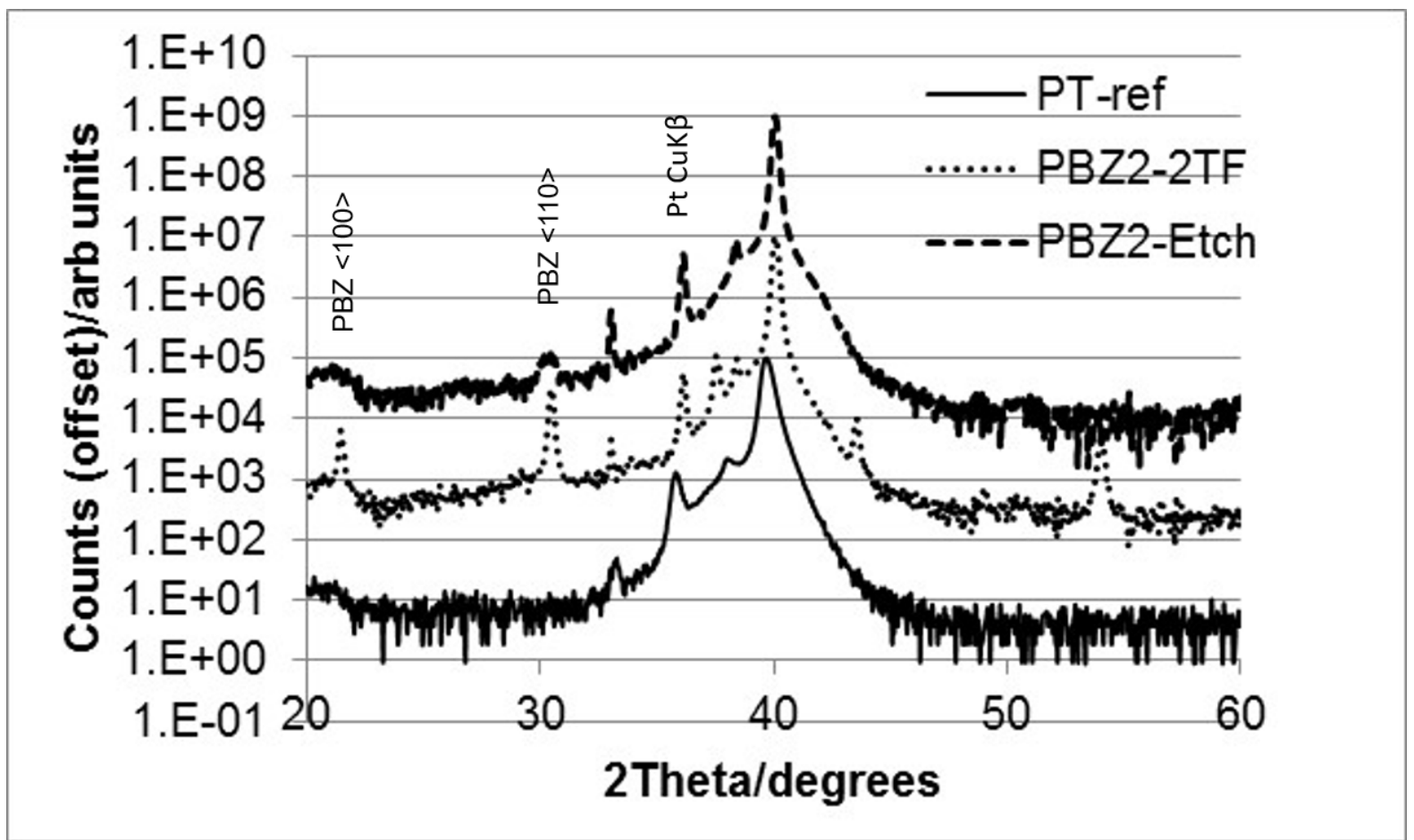

Figure 7. XRD comparison of annealed PBZ and residual films. Difference in XRD before and after wet etching of a PBZ film. 
a)

b)

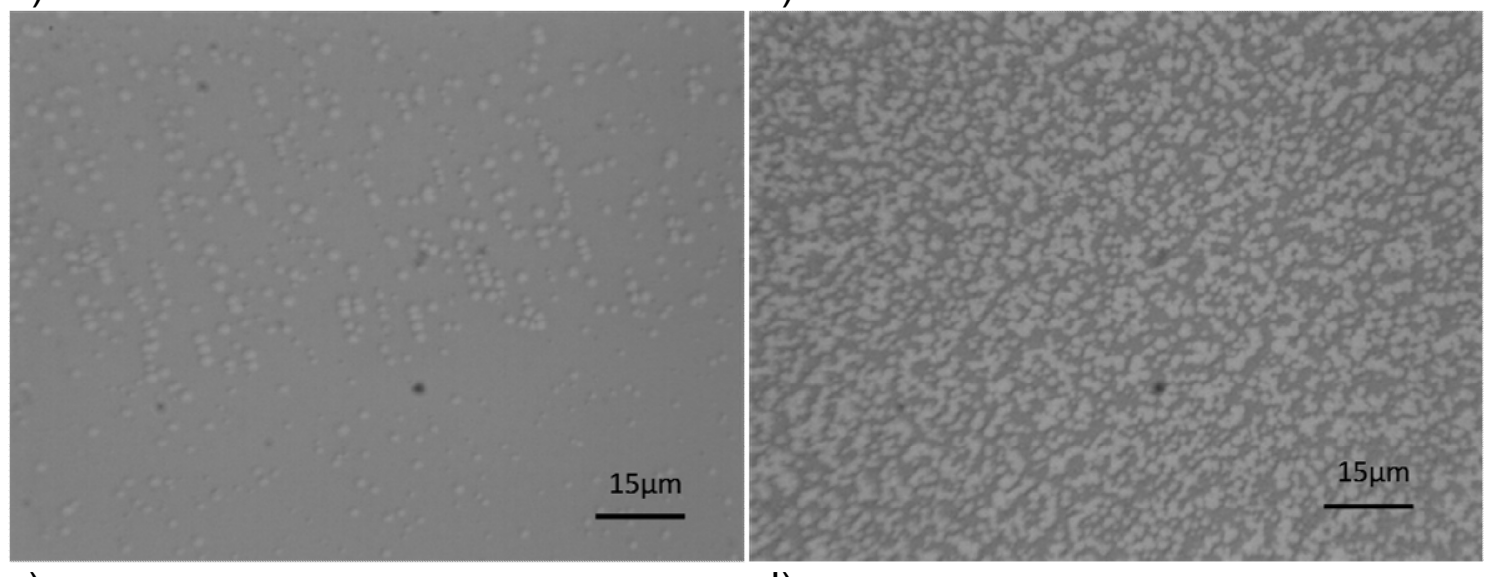

c)

d)

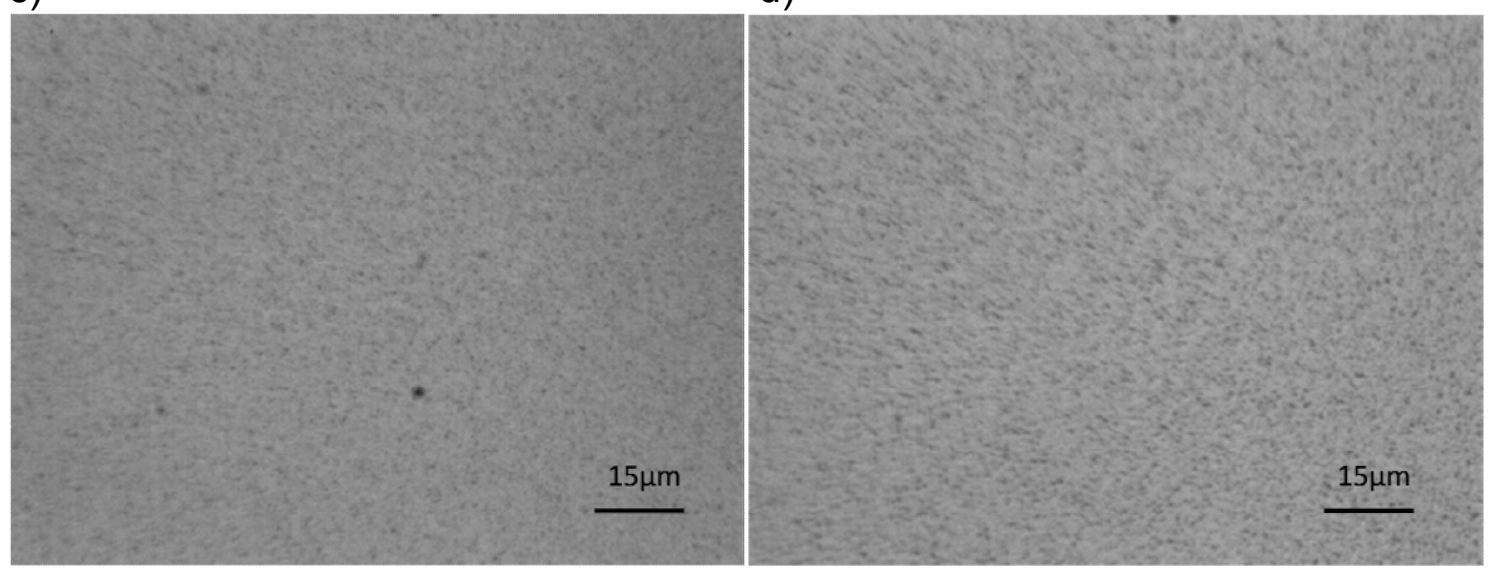

Figure 8. Optical images showing seeding density effects from different hotplate annealing temperature. a) $550^{\circ} \mathrm{C}$. b) $600^{\circ} \mathrm{C}$. c) $650^{\circ} \mathrm{C}$,.d) $675^{\circ} \mathrm{C}$.

Figures 8a-d highlight the influence of the hotplate annealing temperature on the seeding density effects and how that impacts on the film's morphology at this stage of the processing. Further investigation of the PBZ crystallised and etched film morphology is shown by the SEM images in figure 9a-b, whilst table 1 shows EDX findings carried out to look at potential composition variation within the films.

a)

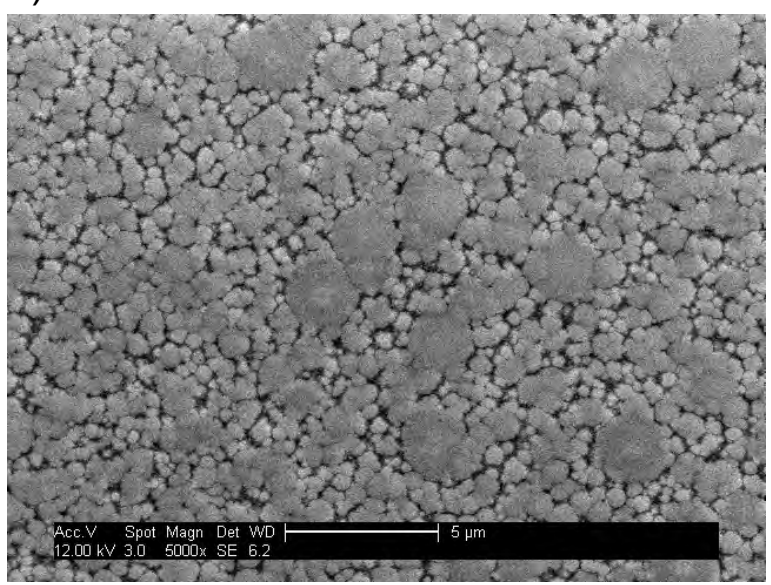

b)

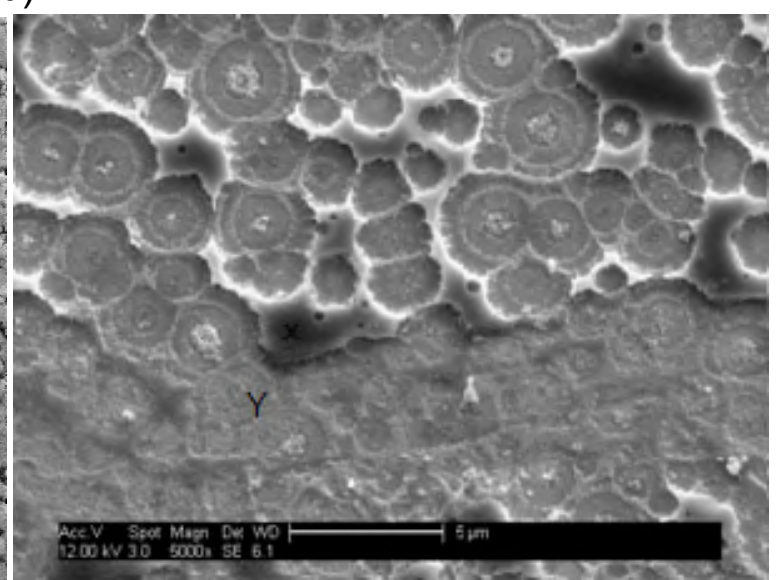

Electrocaloric Effect(ECE) 
Figure 9. Surface SEM images of: a) Annealed PBZ film $\left(550^{\circ} \mathrm{C}(\mathrm{HP}) / 750^{\circ} \mathrm{C}\right.$ furnace $)$. b) Residual film after wet etch. (Area $X$ and $Y$ represent EDX measurements recorded in Table 1).

Table 1. EDX elemental analysis of annealed PBZ film and residual deposit from wet etch.

\begin{tabular}{ccccc}
\hline \multicolumn{5}{c}{ Annealed(atm\%) } \\
\hline (figure 9a) & $\mathbf{P b}$ & $\mathrm{Ba}$ & $\mathbf{Z r}$ & \\
\hline $\begin{array}{c}\text { Average(Whole } \\
\text { area) }\end{array}$ & 14.2 & 3.33 & 13.7 & \\
\hline \multicolumn{5}{c}{ Etched(Atm\%) } \\
\hline (figure 9b) & $\mathbf{Z r}$ & $\mathbf{P t}$ & $\mathbf{O}$ & $\mathbf{S i}$ \\
\hline Island(Area X) & 30.4 & 19.1 & 50.5 & - \\
\hline Rossette (Y) & 0 & 83.1 & 0 & 16.9 \\
\hline $\begin{array}{c}\text { Average(whole } \\
\text { area) }\end{array}$ & 0 & 63 & 21.4 & 11 \\
\hline
\end{tabular}

Figures 10 and 11 show electrical characterisation of the films in terms of the variation of dielectric constant with film thickness (figure 10) and hysteresis (P/E loop) measurements (figure 11).

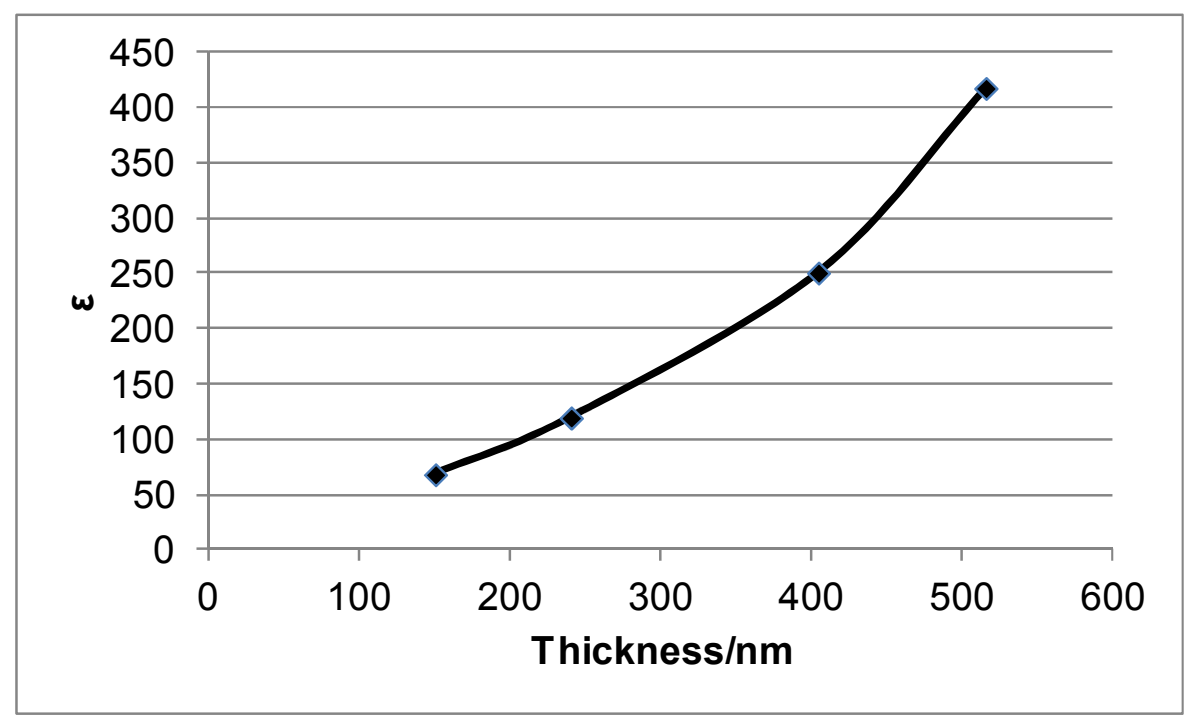

Figure 10. Dielectric Constant vs. PBZ film thickness. Capacitance measured on films of different thickness annealed by $550^{\circ} \mathrm{C} / 750^{\circ} \mathrm{C}$ hotplate/ box furnace processing. 


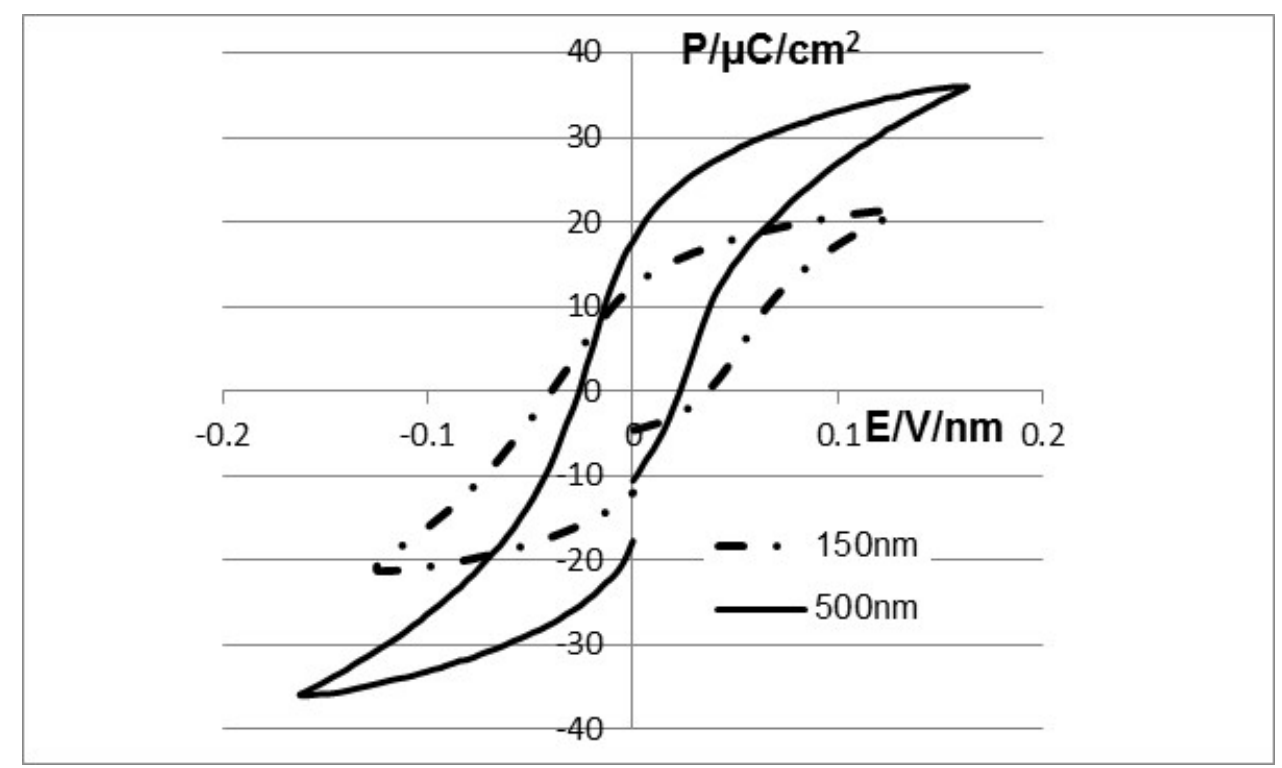

Figure 11. Hysteresis measurements on PBZ films of different thickness.

\section{Discussion}

The overall analysis carried out in this work has highlighted a major issue in trying to recreate the findings from previous studies on PBZ thin films. Based on work by Pokharel et al. [16] which looked at the phase transitions in bulk Pb1-x Bax ZrO3 for $x<0.35$, the presence of antiferroelectric (AFE), ferroelectric (FE) and paraelectric (PE) phases at room temperature depending on the Ba content has been shown. Subsequent studies centred on $x=0.2$ [15] had demonstrated the coexistence of AFE and FE phases at RT close to the AFE-FE phase boundary reported by Pokharel et al. [16] and were attributed as contributing to the potential large electrocaloric effect observed. The XRD analysis based on figure 2 seems to be in agreement with the Peng et al. [15] study in terms of the annealing temperature to remove pyrochlore, but evidence for coexistence of phases has not been observed. In particular, there is no evidence in figure 2 of the $<130 / 112>$ reflections at $27.5^{\circ}$ reported in the previous work which was the only assigned peak that was not coincidental with the expected FE perovskite peaks, at 100,110 and 111 and would help to definitively identify the AFE phase in the present study. In fact the peak identified as rhombohedral ferroelectric (RFE)(-111), might be RFE(111) and the reflection at $38.4^{\circ}$ possibly relates to the shift in a Pt-ref peak after annealing or at least is obscured by this shift. However, work by Bongkarn et al.[17] which looked at a PBZ composition $(x=0.025)$, shows the $X R D$ for the material in a purely AFE phase region and which shows the $202 / 042$ at approximately $37.5^{\circ}$, and the $230 / 212$ at $38.4^{\circ}$, so when this is coupled with the TEM and electron diffraction supporting evidence in the Peng et al. work, the conclusion of a 2 phase system seems justified. This therefore leads onto the question; why the evidence of 2 phases has not been observed in the present work considering the synthesis route was based on the previous reported route. Although figure 5a appears to show two visual morphologies in terms of distinct large rosettes surrounded by smaller structures this is not proof of two coexisting phases. The XRD in figure 2 for the fully annealed films support the presence of an FE single phase which would concur with the evidence from Pokharel et al [16] which suggests the AFE-FE phase transition for a composition of $x=0.20$ is 
near $5^{\circ} \mathrm{C}$. However, it could be the case that only a small difference in compositional variation is required to raise the phase transition closer to RT, particularly in this "steep" region of the phase boundary and start to develop a mixed phase. Such variation could be possible from errors in initial weighing, precipitation during sol synthesis (as had been observed in initial sol synthesis) or from varying amounts of lead loss during annealing. Another aspect of annealing thin films has always been the preferential growth of films dependent on the substrate and temperature profile, and that this can also have a significant effect on the film morphology. The "large" rosette structure shown in figure $5 \mathrm{~b}$ of an annealed PBZ film has been shown before for both PBZ[15] and lead zirconate films [18]. In this particular case it can also be seen that from figure $5 a$ where the film was at the prefurnace stage, there is visual evidence of what may be the seeding centres for the final rosette structures. Similar observations to this for PZT growing on ITO coated substrates where the annealing temperature is too low (or annealing time too short), have been previously reported [19]. Further development of the effects of temperature profile on film morphology are shown in figures 8a-d which summarises the optical images for films only annealed on hotplates. Here, the increase in seeding density of the rosettes with annealing temperature between $550^{\circ}$ and $675^{\circ} \mathrm{C}$ can readily be seen, which subsequently impacts on the achievable rosette size observed in the finally annealed films. Of course the heating rate of annealing also plays an important role in the observed film morphology. Figure $8 \mathrm{~d}$, showing a sample annealed rapidly to $675^{\circ} \mathrm{C}$ on a hotplate, displays the small, high density, rosette morphology, where as figure $5 \mathrm{~b}$, which was annealed in a furnace to a higher temperature $\left(750^{\circ} \mathrm{C}\right)$ but slowly, retains the rosette structures evident at the intermediate temperature step $\left(550^{\circ} \mathrm{C}\right)$, but with a finer texture between the large structures. In terms of preferential crystal growth indications, where a particular orientation is dominant often as a result of substrate influences a number of particular trends can be seen in the XRD analysis. Typically for a random orientation XRD pattern of perovskite the 101 reflection at $30.5^{\circ}$ is found to be larger than the 100 or 111 reflections, but it has been seen in the past that effects from the Pt substrate such as the formation of intermetallic phases with lead [20] can favour the 111 orientation of the film. Figure $3 a$ and $3 b$ show that some 111 orientation was favoured for samples annealed in the small diameter tube furnace, whereas the XRD of the larger box furnace samples were more typical of a random spectra. Figure 2 does have one example (600HP/750BF) which shows a preferential 111 orientation using the box furnace, but it is worth noting that the intermediate hotplate temperature was $600^{\circ} \mathrm{C}$, and the only other trace in figure 2 which shows a similar 111 orientation is that annealed on a hotplate at $650^{\circ} \mathrm{C}$. This suggests that hotplate annealing above $600^{\circ} \mathrm{C}$ will induce the 111 preferential orientation prior to the furnace treatment, but for $550^{\circ} \mathrm{C}$, it was only induced using the small tube furnace. This finding may be useful for the scale up to in a full wafer processing scenario, as an increase in the initial annealing temperature may be a practical solution to achieve a particular crystal orientation, when having to use the box furnace to accommodate a larger sample size. One potential reason for the effect could lie in the different heating rates of the two furnaces, where the tube furnace ramped up at on average $1.3^{\circ} \mathrm{K} / \mathrm{s}$ in the critical $475-750^{\circ} \mathrm{C}$ region compared to only $0.7^{\circ} \mathrm{K} / \mathrm{s}$ for the box furnace. Alternatively, the difference in the geometry of the heating coils in the two furnaces, with the box furnace only having them above the sample compared to a more "all-round" arrangement for the tube furnace might 
encourage a substrate influence which in the past has been shown to drive the 111 growth.

Another important observation from this study has been the analysis of the residue left after the wet etching of the PBZ films that was carried out to access the $\mathrm{Pt}$ electrode for electrical characterisation. The presence of a precipitate ( $\mathrm{PbCIF}$ ) has been known to form during the $\mathrm{HF} / \mathrm{HCl}$ step of the wet etch of PZT[21], which has lead to the inclusion of a second HNO3 step to dissolve the precipitate. However, even relatively prolonged soaking in the $\mathrm{HNO} 3$ could not dissolve the residue observed from these PBZ films. The SEM image in figure 9a shows a fully annealed film, which still contains the two apparently distinct growth morphologies observed in the optical images shown in figures 6 and 7. Certainly large rosette structures stand out, $\sim 2$ micron in diameter, which relate to the early features that were visible after just hot plate annealing at $550^{\circ} \mathrm{C}$ (figure $5 \mathrm{a}$ ). These seem to be initial seeding points of the films, and the subsequent finer rosette structures ( $<1$ micron) which can be seen between them develop during the furnace anneal at a higher seeding density due to the higher temperature. It's possible that these two different sized rosettes are different in composition due to the film composition at the point they start to seed, but it is believed that they are both a composition containing $\mathrm{Pb} / \mathrm{Ba} / \mathrm{Zr}$. Figures $6 \mathrm{a}-$ $b$ (optical) and figure 9b (SEM) show that after etching, a skeletal structure remains which mimics the original morphology of the annealed film. This structure was very weak and easily disturbed as shown by the smear marks on figure $6 \mathrm{~b}$. Figure $9 \mathrm{~b}$ (SEM) particularly emphasises how the residue is concentrated in the "island" regions where the enlarging rosettes don't manage to coalesce, and also as a small annulus around the centres of large rosettes. Figure 7 shows the XRD comparison for annealed and etched samples, and shows that a broad residual peak still exist at $30.5^{\circ} \mathrm{C}$ in the etched sample, but the supporting EDX analysis carried out on the samples and based on figures $9 a-b$ and table 1 indicate that the only metal remaining from the original film composition is zirconium. Of course it's important to remember that the precipitate from the wet etch process is not necessarily a compound that was present in the annealed film (eg.like the PbCIF in the PZT case) but a biproduct. However, the fact that no lead or barium is observed in the residue of the PBZ etched films combined with in the PZT example where no residue is seen at all after the 2 step etch, this could be evidence of some of the zirconium existing in this study in a non-PBZ phase, and figures $6 \mathrm{~b}$ or $9 \mathrm{~b}$, can be viewed as "sample maps" of where this non-PBZ phase existed. The large variation in atomic percentage seen in table 1 and obtained from the two distinct regions analysed $(X$ and $Y$ ) of figure $9 \mathrm{~b}$ can be attributed to the distribution of the proposed non-PBZ phase. In PBZ regions (rosette structures), the $\mathrm{Pb} / \mathrm{Ba} / \mathrm{Zr}$ atoms are intimately combined so that on etching, all the component atoms are removed and wash away. In the island areas $(\mathrm{X})$ which are the proposed non-PBZ regions, a resistant $\mathrm{Zr}$ containing structure exist which withstands the etching process. The apparent lack of $\mathrm{Zr}$ for the whole area of figure $9 \mathrm{~b}$ even though it was observed for area $\mathrm{X}$ is simply related to the scale of the relevant areas analysed (1000x difference), which hugely dilutes the $\mathrm{Zr}$ signal relative to the background substrate.

The phenomenon of developing rosettes in PZT films on ITO mentioned previously [19] was proposed in terms of a $\mathrm{Pb}$ diffusion towards pyrochlore regions as the crystallization process proceeded, and which developed an observed central peak to the rosette structures. Whether a similar mechanism in terms of Pb diffusion can be suggested in the PBZ case is difficult to say because of the different substrate 
present. However it is interesting that a central feature remains in figure $9 \mathrm{~b}$ after etching, although as indicated above its composition is not linked to pyrochlore. Looking at table 1 for the annealed film, where the average value is taken over the whole image shown in figure $9 \mathrm{~b}$, which should allow for local variations in composition, the relative ratios of $\mathrm{Pb}: \mathrm{Ba}: \mathrm{Zr}$ of 1.04:0.24:1 compared to the target 0.8:0.2:1 are significantly different. Even considering the initial sol composition contained $20 \%$ excess $\mathrm{Pb}$, so the as-spun deposited ratio should have been $0.96: 0.2: 1$, these values still show the $\mathrm{Pb}$ content less than the $\mathrm{Zr}$, and would be expected to decrease due to Pb-loss during annealing. Of course, the effect of not knowing the exact $\mathrm{Pb}$-loss, complicates the quantitative comparison of the ratio values, but one potential cause of the discrepancy could be linked to a loss of $\mathrm{Zr}$ during the spinning stage as a consequence of the non-sol nature of the deposited solution. This potentially could also be linked to the non-PBZ phase referred to above. In a sol, there are bridging links between the constituent metal compounds, so that when the materials are deposited the correct proportion of constituents will be present in local environments which facilitates the formation of the target composition. For the case of a solution, where the bridging links are not developed, there is the potential during the spin/drying stage that a non uniform distribution in terms of the constituents might be deposited. For example, during spinning, a proportion of the solution does not coat the substrate and is spun off. In the critical spin/drying phase as the components start to gel/deposit from the solution case, differing solubilities may then effect the composition deposited. In this particular case, the evidence might suggest that a loss of $\mathrm{Zr}$ is occurring during this step as shown by the enhanced level of $\mathrm{Pb}$ in the final films. Either way, a non-uniform phase morphology can develop across the substrate on annealing. Another potential reason for not observing the mixed AFE/FE phases may be related to the initial purity of starting reagents providing small levels of doping. Small variations in composition in the region of expected phase boundaries by impurity doping can have significant effects on observed properties. Consequently a comparison of the purity of reagents in other studies may be needed to justify this conclusion, although the observation of the proposed $\mathrm{ZrO} 2$ phase is a more significant concern regards compositional change.

Table 2. Optimised electrical properties of annealed PBZ film. (Thickness-515nm, annealed $350^{\circ} \mathrm{C}-\mathrm{HP}(3 \mathrm{~min}) / 550^{\circ} \mathrm{C}-\mathrm{HP}(5 \mathrm{~min}) / 750^{\circ} \mathrm{C}(30 \mathrm{~min})$-Dielectric properties measured at $1 \mathrm{kHz}$.)

\begin{tabular}{cccc}
\hline$\varepsilon$ & Tan $\delta$ & $\begin{array}{c}\text { Film Resistivity } \\
/ \Omega-m\end{array}$ & $\begin{array}{c}\text { Shorting } \\
\text { Field } \\
\text { MV/m }\end{array}$ \\
\hline 400 & 0.02 & $8 \times 10^{8}$ & 90 \\
\hline
\end{tabular}

Although an extensive electrical characterisation is not presented in this study and the values given in Table 2 represent the optimum values obtained in this study, the drive to modify the crystallization path for the PBZ films was initiated by poor dielectric properties obtained for the early four layer films whose XRD were shown in figure 2. The work by Peng et al. [15] had reported dielectric constants in the region of 550 and loss values (tanס) around 0.02 , and although the loss figures were Electrocaloric Effect(ECE) 
matched, a dielectric constant of between only 180-270 for the initial thinner films was found. Figure 10 shows a comparison of relative permittivity $\left(\varepsilon_{r}\right)$ vs film thickness compiled from samples in this study, and shows $\varepsilon_{r}$ increasing with thickness. A similar study reported by Habouti et al. [22] on PZT which was looking at the interface effect on the measured capacitance for different thicknesses shows what appears to be an opposite trend to that observed in this study. An increase in thickness of a film with the same dielectric constant would produce a decreasing capacitance $(C)$ with film thickness according to, $C=\varepsilon_{0} \varepsilon_{1} A / s$, where $s_{0}$ is the permittivity of free space, $\varepsilon_{r}$ is the relative permittivity or dielectric constant, $A$ is the area of the file, and $s$ its thickness. A potential explanation for this could be linked to the proposed Zirconium(non-PBZ) phase being present in the films. The SEM image in figure $9 \mathrm{~b}$ shows the residue pattern mimicking the original morphology structure which suggests this particular phase was connected to the Pt electrode when it was formed. Thicker samples are formed by the deposition of more layers and the lower layers effectively experience longer pre annealing times. Evidence has already been shown in this study how the pre anneal conditions can affect the subsequent post furnace annealed film state and this would impact on the dielectric film properties. This could produce an area or volume effect where the PBZ was depositing as pillars from seed points that grow in and out of plane at the same time. In this case the area as well as the thickness of the high dielectric constant PBZ material would increase. If the radial growth $(r)$ as well as the thickness $(s)$ was proportional to the anneal time $(t)$ through proportionality constants $a$ and $b$ respectively, so $r=a t$ and $s=b t$, then the capacitance would depend on anneal time so $C=\varepsilon_{0} \varepsilon_{r} \pi a^{2} t^{2} / b t$ or $C=\varepsilon_{0} \varepsilon_{r} \pi a^{2} t / b$ giving the observed increase of capacitance with growth time and film thickness. The idea of PBZ pillar growth is not dissimilar to the observed morphology from the SEM images. The main difference of the samples deriving the data for the different thicknesses in figure 10, is the number of intermediate annealing steps at 350 and $550^{\circ} \mathrm{C}$. It is likely that during the $550^{\circ} \mathrm{C}$ hot plate anneals the development of PBZ seed centres will occur and that in the thicker films will be greater in number or more advanced going into the same furnace anneal that all the samples experience. Couple this with the presence of the lower dielectric constant $\mathrm{Zr}$ phase which would represent a greater volume of thinner films than thicker ones (assuming it remains constant) then the potential for increased capacitance with film thickness would be possible. Another consequence of multiple annealing times for the lower layers as film thickness increases is that this allows for potential increased grain and domain growth to occur, which can give the potential for increases in polarisation contribution. So it may be the case that for the thinner films, the length of annealing time may not have been the optimum. In addition, the amount of lead loss from thin films subjected to different annealing times could also play a contributing role. Figure 11 also suggests there is some form of incomplete development within processed films of different thickness, as indicated by the variation in coercive field for the different films. Figure 11 shows that the thicker PBZ film has a narrower PE-loop and requires a smaller coercive field than the thinner sample. This again may be linked to the presence of the proposed Zr-phase and its ratio to the PBZ component depending on the overall anneal time as outlined above, and the subsequent effects this has on electrical properties of the sample as a whole. The profile of both the loops which are characteristic of a ferroelectric phase and don't show any double loop nature, also helps to support the XRD observations of no apparent AFE phase within the films studied. 


\section{Conclusions}

The work covered in this paper has primarily concentrated on the deposition and characterisation aspects of $\mathrm{Pb0.8Ba0.2ZrO3}$ thin films, based on their potential use in applications utilising the electrocaloric effect as previously reported. The synthesis of solutions for spin coating that showed a stable shelf life of over 6 months was demonstrated, but it has been proposed that this stability may be counterproductive in achieving the target composition in finally annealed films. Compositional studies of annealed films suggested that the $\mathrm{Zr}$ content was lower than expected, with a proposal that this was being lost at the spin coating stage due to the non-sol nature of the solution. In addition to this, the observation of an insoluble, residual, skeletal, structure resulting during wet etching, and mimicking the original annealed rosette morphology showed only the presence of $\mathrm{Zr}$ from the potential $\mathrm{Pb} / \mathrm{Ba} / \mathrm{Zr}$ metal elements of the sol. This suggested a non-PBZ phase was also present in the annealed films although not observed by XRD, and was put forward as contributing to the low dielectric constant. Previously reported coexisting AFE and FE phases within the annealed films were not observed in this study, but proposed reasons linked to the close proximity of the AFE-FE phase transition at room temperature and potential causes of compositional change in this region were put forward. However, in the drive to optimise dielectric properties, important findings in relation to crystallization temperature, type of furnace used and preferred film growth, were observed which will be of use when incorporating the films within potential electrocaloric test devices. It may still be the case however, that the resultant films require the mixed AFE and FE phase to demonstrate the desired electrocaloric magnitude.

\section{Acknowledgements}

The authors would like to acknowledge support from the European Metrology Research Programme (EMRP) (jointly funded by the EMRP participating countries within EURAMET and the European Union) and the UK National Measurement System.

\section{References}

1. Valant M, Electrocaloric materials for future solid-state refridgeration technologies. Progress in Materials Science 2012;57:980-1009.

2. Parui J, Krupanidhi SB. Electrocaloric effect in antiferrroelectric $\mathrm{PbZrO} 3$ thin films. Phys Status Solidi 2008;2(5):230-2.

3. Hao X, Yue Z, Xu J, An S, Nan C-W. Energy-storage performance and electrocaloric effect in (100)-oriented $\mathrm{Pb}_{0.97} \mathrm{La}_{0.02}\left(\mathrm{Zr}_{0.95} \mathrm{Ti}_{0.05}\right) \mathrm{O}_{3}$ antiferroelectric thick films. J Appl Phys 2011;110:064109.

4. Chukka R, Cheah JW, Vhen Z, Yang P, Shannigrahi S, WangJ, et al. Enhanced cooling capacities of ferroelectric materials at morphotropic 
phase boundary. Appl Phys Lett 2011;98:242902.

5. Saranaya D, Chaudhuri AR, Parui J, Krupanidhi SB. Electrocaloric effect of PMN-PT thin films near morphotropic phase boundary. Bull Mater Sci 2009;32(3):259-62.

6. Feng Z, Shi D, Dou S. Large electrocaloric effect in highly (001)-oriented $0.67 \mathrm{PbMg}_{1 / 3} \mathrm{Nb}_{2 / 3} \mathrm{O}_{3}-0.33 \mathrm{PbTiO}_{3}$ thin films. Solid State Commun. 2011;151:123-26.

7. Correia TM, Kar-Narayan S, Young JS, Scot tJF, Mathur ND, Whatmore RW, et al. PST thin films for electrocaloric coolers.J Phys D, Appl Phys 2011;44:165407.

8. He Y, Li XM, Gao XD, Leng X, Wang W. Enhanced electrocaloric properties of PMN-PT thin films with LSCO buffer layers. Funct. Mater. Lett. 2011;4(1):45-8.

9. Mischenko AS, Zhang Q, Whatmore RW, Scott JF, Mathur ND. Giant electrocaloric effect in the thin film relaxor ferroelectric $0.9 \mathrm{PbMg}_{1 / 3} \mathrm{Nb}_{2 / 3} \mathrm{O}_{3}-$ $0.1 \mathrm{PbTiO}_{3}$ near room temperature. Appl Phys Lett 2006;89:242912.

10. Correia TM, Young JS, Whatmore RW, Scott JF, Mathur ND, Zhang Q. Investigation of the electrocaloric effect in a $\mathrm{PbMg}_{2 / 3} \mathrm{Nb}_{1 / 3} \mathrm{O}_{3}-\mathrm{PbTiO}_{3}$ relaxor thin film. Appl Phys Lett 2009;95:182904.

11. Feng Z, Shi D, Zeng R, Dou S. Large electrocaloric effect of highly (100)oriented $0.67 \mathrm{PbMg}_{1 / 3} \mathrm{Nb}_{2 / 3} \mathrm{O}_{3}-0.33 \mathrm{PbTiO}_{3}$ thin films with a $\mathrm{Pb}\left(\mathrm{Zr}_{0.3} \mathrm{Ti}_{0.7}\right) \mathrm{O}_{3} / \mathrm{PbOx}$ buffer layer. Thin Solid Films 2011;519:5433-6.

12. Chen H, Ren TL, Wu XM, Yang Y, Liu LT. Giant electrocaloric effect in lead-free thin film of strontium bismuth tantalite. Appl Phys Lett 2009;94:182902.

13. Lu SG, Rozic B, Zhang QM, Kutnjak Z, Pirc R, Lin M, et al. Comparison of directly and indirectly measured electrocaloric effect in relaxor ferroelectric polymer. Appl Phys Lett 2010;97:202901.

14. Mischenko AS, Zhang Q, Scott JF, Whatmore RW, Mathur ND. Giant electrocaloric effect in thin-film PbZr0.95Ti0.0503. Science 2006;31(1):1270-1.

15. Peng $B$, Fan $H$, Zhang Q. A giant electrocaloric effect in nanoscale antiferroelectric and ferroelectric phases coexisting in a relaxor $\mathrm{Pb0.8Ba0.2ZrO3}$ thin films at room temperature. Adv. Funct. Mater. 2013;23: 2987-92.

16. Pokharel BP, Pandey D. Dielectric studies of phase transitions in (Pb1xBax)ZrO3. J. Appl. Phys. 2000;88:5364-73.

17. Bongkarn T, Vittayakorn N. Effect of Excess $\mathrm{PbO}$ on Phase Formation, Microstructure and Dielectric Properties of (Pb0.975Ba 0.025)ZrO3 Ceramics. NU Science Journal 2007; 3(2):111-17.

18. Chen SY, Chen IW. Temperature-Time Texture Transition of $\mathrm{Pb}(\mathrm{Zr1} 1-\mathrm{xTix}) \mathrm{O} 3$ Thin Films: II, Heat Treatment and Compositional Effects. J. Am. Ceram. Soc. 1994;77(9):2337-44.

19. Roy SS, Gleeson H, Shaw CP, Whatmore RW, Huang Z, Zhang Q, et al. Growth and characterisation of lead zirconate titanate (30/70) on indium tin oxide coated glass for oxide ferroelectric-liquid crystal display application. Integrated Ferroelectrics 2000:29(3-4);198-213. 
20.Zhang Q, Huang Z, Vickers ME, Whatmore RW. Effect of the particle size in PZT precursor sols on the orientation of the thin films. J. of European Ceramic Society. 1999;19:1417-21.

21. Zheng $\mathrm{K}$, Lu J, Chu J. A novel wet Etching process of $\mathrm{Pb}(\mathrm{Zr}, \mathrm{Ti}) \mathrm{O} 3$ thin films for applications in microelectromechanical system. Japanese Journal of Applied Physics 2004;43(6b):3934.

22. Habouti S, Lahmar A, Dietze M, Solterbeck C, Zaporojtchenko V, Es-Souni M. Substrate heterostructure effects on interface composition, microstructure development and functional properties of PZT thin films. Acta Materialia 2009;57:2328-38. 


\section{Characterisation of lead barium zirconate thin films for utilisation of the electrocaloric effect}

Shaw, Christopher P.

Elsevier

C.P. Shaw, Qi Zhang, T.M. Correia, P.M. Weaver, Characterisation of lead barium zirconate thin films for utilisation of the electrocaloric effect, Materials Chemistry and Physics, Volume 178, 1 August 2016, pp74-81

http://dx.doi.org/10.1016/j.matchemphys.2016.04.070

Downloaded from Cranfield Library Services E-Repository 